\title{
Contribuição ao estudo do gênero Vismia Vandelli (Guttiferae)
}

\author{
Maria Elisabeth van den Berg (") \\ Museu Paraense Emilio Goeldi
}

\section{RESUMO}

Notas taxonômicas sobre Vismia cayennensis (Jacquir) Persoon, V. subcuneata Huber e V. cavalcantii van den Berg, incluindo a nova variedade Vis. mia cayennensis (Jacquin) Persoon var. tenuinervia van den Berg e a nova combinação Vismia cayenensis (Jacquin) Persoon var. sessilifolia (Aublet) van den Berg.

\section{INTRODUÇÃo}

O exame das coleções de Vismia cayennensis (Jacquin) Persoon, pertencentes aos herbários do Museu Paraense Emílio Goeldi (MG), Instituto de Pesquisas e Experimentação Agropecuárias do Norte (IAN), Instituto Nacional de Pesquisas da Amazônia (INPA), Jardim Botânico do Rio de Janeiro (RB) e Museu Nacional $(R)$, além de coletas avulsas, indicam a existência de uma variedade nova bem caracterizada, que é descrita neste trabalho.

As espécies $V$. sessilifolia (Aublet) DC., $V$. ramuliflora Miquel e $V$. rufescens (Lamarck) Persoon são bastante controvertidas e o seu estudo demonstra que se trata de um único taxon, constituindo variedade de V. cayennensis, sendo necessária uma nova combinação.

Ewan (1962) considera V. subcuneata Huber apenas uma subespécie de $V$. baccifera (L.) Triana et Planchon, o que é analisado neste trabalho.

Em 1970, van den Berg descreveu V. cavalcantii registrando-se novas ocorrências na Amazônia.

\section{I - Vismia cayennensis (Jacquin) Persoon}

O grande número de coleções examinadas (cerca de duzentas) permite aquilatar-se a variabilidade desta espécie, que não é das maiores se comparada às outras espécies do gênero. Entretanto, além da variedade que abrange o "typus", mais duas variedades são inconfundiveis, sendo uma nova e outra resultante de uma nova combinação, baseada nos resultados de uma revisão das espécies $V$. sessilifolia (Aublet) DC., V. ramuliflora Miquel e V. rufescens (Lamarck) Persoon. Ewan (1962) considerou estas duas últimas sinônimos da primeira, mantendo, entretanto, V. sessilifolia como espécie válida. Está confirmado que as citadas espécies constituem o mesmo taxon, sendo, porém, apenas uma variedade de V. cayennensis o que exige uma nova combinação.

\section{CHAVE DE SEPARAÇÃO ENTRE VARIEDADES DE V. CAYENNENSIS}

1. Lâmina foliar com ápice subcaudato, com 20-28 pares de nervuras secundárias muito delicadas. Pedúnculos florais ultrapassando $11 \mathrm{~mm}$ de comprimento. ..... var. tenuinervia

1. Lâmina foliar com ápice agudo ou acuminado, com 5-13 pares de nervuras secundárias robustas. Pedúnculos florais com $4-7 \mathrm{~mm}$ de comprimento.

2. Lâmina foliar cartácea, de $4,5-9 \mathrm{~cm}$ de comprimento e $3,5-4,5 \mathrm{~cm}$ de largura. Inflorescências corimbiformes...... var. cayennensis

(") - Bolsista do Conselho Nacional de Pesquisas 
Lâmina foliar coriácea, de 10-15 (até 19) $\mathrm{cm}$ de comprimento e $5-9,5 \mathrm{~cm}$ de largura. Inflorescências glomeruliformes ............ $\ldots \ldots \ldots \ldots . . . \ldots \ldots \ldots$ var. sessilifolia

Vismia cayennensis (Jacquin) Persoon var. tenuinervia van den Berg var. nov.

Apicem folii subcaudato; 20-28 paribus nervorum secundariorum tenuiorum, 1,5-2,5 $\mathrm{mm}$ distantium inter se. Pedunculis floralibus 11-19 mm longis.

HOLCTYFUS: BRASIL: Pará: Acará, Jacarequara. tapera, "apui"; 20-II-1966; M. Silva 501 (MG).

MATERIAL ADICIONAL: BRASIL: Pará: Rio Moju, Fábrica; 31-V-1954; G. A. Black 54-16236 (IAN). Gurupá; 24-II-1923; A. Ducke (RB 18066).

Esta variedade é facilmente distinta pela lâmina foliar subcaudada, nervuras muito regularmente emparelhadas e pedúnculos florais longos que dão à inflorescência um aspecto gracioso. Os botões florais apresentam-se mais robustos, com cerca de $6 \mathrm{~mm}$ de altura e $5 \mathrm{~mm}$ de diâmetro.

Vismia cayennensis (Jacquin) Persoon var. sessilifolia (Aublet) van den Berg comb. nov.

Hypericum sessilifolium Aublet PI. Guian. 2:787. tab. 312 ,fig. 2. 1775.

H. rufescens Lamarck Encycl. 4:150. 1796. Typus: Arcura, Guiane Française; 17-21-XIStoupy (P. Photo FM 35239!).

Vismia rufescens Persoon Syn. PI. 2:86. 1806.

V. rufescens var. sessilifolia Persoon I.c.

V. sessilifolia (Aublet) DC. Prod. 1:542. 1824.

V. ramuliflora Miquel Stirp. Surinam.: 88. 1850. Typus: "Sylvis umbrosis regionum interiorum" Surinam; IV-1847; Hostmann \& Kappler 1823 (holotypus U, isotypus G,S; Photo FM 35238 de um espécime de P!).

Caopia sessilifolia Kuntze Rev. Ge. PI. 1/59. 1891.

C. sessilifolia var. rufescens Kuntze I.c.

Typus: "Cayenne", Aublet (holotypus BM, Photo NY!. isotypus G-DC).

\section{MATERIAL ESTUDADO E DISTRIBUIÇĀO} GEGGRAFICA:

BRASIL: Estado do Pará: Rio Paru de Oeste, Missão Tiriyó, arredores da Missão - 2020' N 55॰45' W "Subosque dar mata virgem"; 1-III-1970; P. Cavalcante 2576 (MG). - Faro, Campos do Tigre, mata; 31-XII-1919; A. Ducke (RB 12494). Rio Jari, Planalto Monte Dourado; 12-II-1968; N. T. Silva 855 (IAN).

Estado do Acre: Rios Juruá, Cruzeiro do Sul, Estrada da Alemanha, "disturbed ground by stream"; 15-IV-1971; G. T. Prance et alii 11915 (MG, IAN). Rio Moa, "at cachoeira grande, capoeira"; 27-IV-1971; G. T. Prance et alii 12526 (MG. IAN). Seringal Boa Água; VII-1972; J. M. Pires \& N. A. Rosa 13741 (IAN).

Território Federal do Amapá: "bcira do rio Oiapoque"; 31-I-1950; R. L. Fróes 25746 (IAN).

S U R I N A M E : Brownsberg, Arbor n. 101; 10-IX-1917; s/col. (BW 3188, IAN).

Esta variedade distingue-se pelas lâminas foliares coriáceas, bem maiores em relação à var. cayennensis (ver a chave), nervação e reticulação também proporcionalmente maiores em relação var. cayennensis. As inflorescências (panículas glomeruliformes) são predominantemente axilares.

A primeira vista, o aspecto das folhas e das inflorescências desta variedade induzem a considerá-la uma espécie diferente, porém, examinado o material com atenção, são notáveis as caracteristicas típicas de $V$. cayennensis. Aliás, fato este já observado por Reichardt (1878) que colocou V. ramuliflora em sinonímia de $V$. cayennensis o que também não se justifica, por causa das diferenças acima enumeradas.

O epíteto sessilifolia, embora não apropriado, pois, geralmente as folhas desta variedade têm pecíolo conspícuo, ou raro, subsésseis, deve ser conservado, por ser prioritário (Stafleu, 1972).

\section{II - Vismia subcuneata Huber}

Vismia subcuneata Huber foi descrita em 1906, não tendo o seu autor apresentado ilustração, o que é feito neste trabalho. Ewan (1962) considera-a como sub-espécie de $V$. baccifera, fazendo uma nova combinação: Vismia baccifera (L.) Triana et Planchon subsp. subcuneata (Huber) Ewan. 

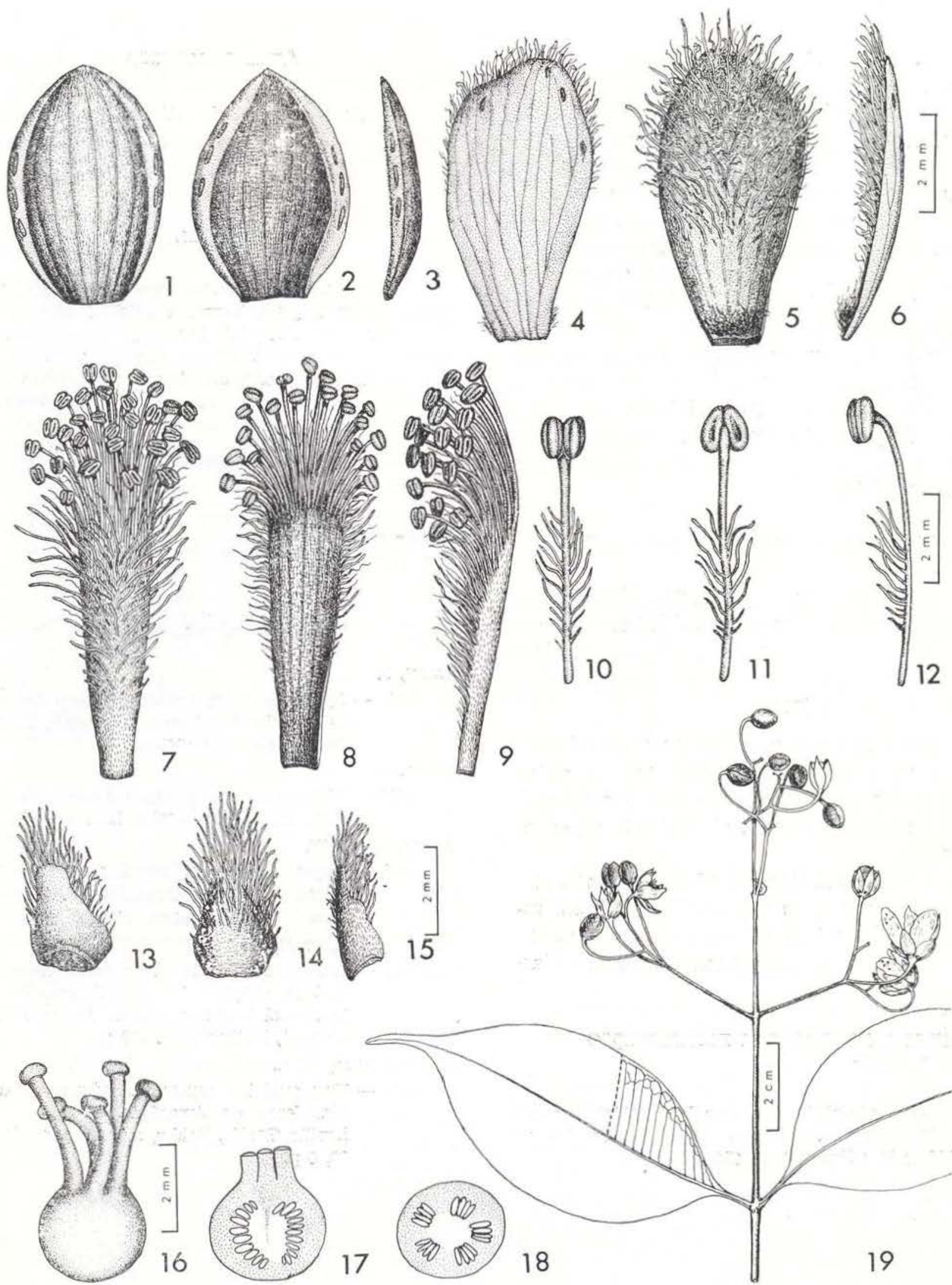

17

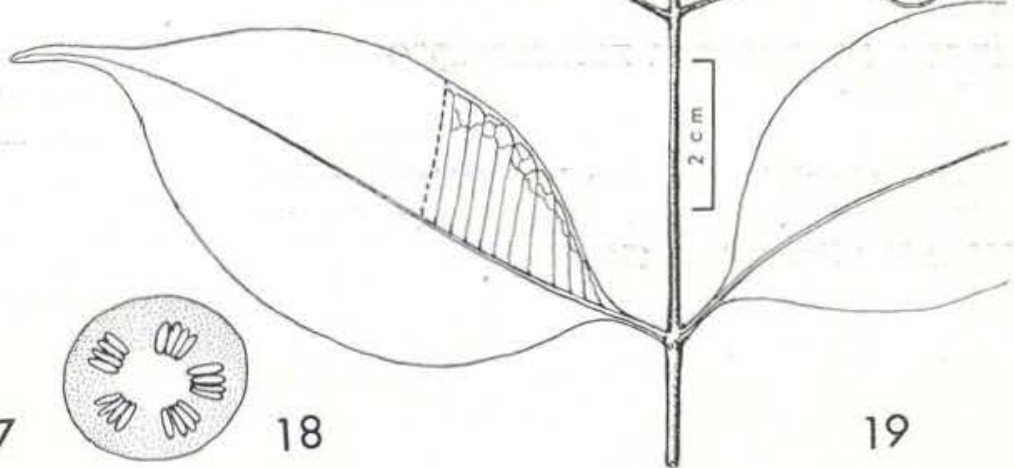

Vismia subcuneata (Holotypus: Huber 1479): 1, 2 e 3) lacinio do cálice em vista externa, interna e lateral, respectivamente; 4.5 e 6 ) pétala. idem; 7,8 e 9) falange de estames, idem; 10 , 11 e 12) estame, idem; 13, 14 e 15) estaminódio, idem; 16, 17 e 18) ovário em aspecto geral, cortes longitudinal e transversal, respectiva. mente; Vismia cayennensis var. tenuinervia; 19) ramo florífero. 
Porém, o estudo do "holotypus" desta espécie (Huber 1479), depositado no Herbário do Miuseu Goeldi, permite a conclusão de que a inflorescência pauciflora e compacta, a pétala de forma obovada ou ovacio-oblonga e assimetricamente truncada da pétala, a forma prismática e assimétrica do estaminódio e a forma capitada do estigma são as principais caracteristicas que tornam $V$. subcuneata diferente de $V$. baccifera. Esta última apresenta inflorescência multiflora, mais aberta, pétala orbicular, ou quando oboval, sempre unguiculada, estaminódio escamiforme retangular e estigma depresso-capitado, lobado, com presença de pelos. As citadas diferenças demonstram a validade de Vismia subcuneata Huber como espécie.

\section{III - Vismia cavalcantii van den Berg}

Duas coleções adicionais, identificadas como Vismia cavalcantii van den Berg var. cavalcantii, portando frutos maduros, ensejam que seja completada a diagnose original baseada em frutos jovens.

Esses frutos são ovaliformes, com 4-6mm de altura e $3-3,5 \mathrm{~mm}$ de diâmetro, coroados com restos de estiletes; o cálice é persistente e fortemente reflexo, circundando o pedúnculo.

A coleção E. Oliveira 1844 apresenta, ainda, lâminas foliares bastante grandes em relação às de outras coleções, algumas alcançando até $9 \mathrm{~cm}$ de comprimento e $3,8 \mathrm{~cm}$ de largura.

\section{MATERIAL ESTUDALO E DISTRIBUIÇĀO CIEOGRAFICA:}

BRASIL: Pará: Rio Paru de Oeste, "Tiriós", campo; 12-V-1962; E. Oliveira 1844 (IAN): Idem; 14.V-1952; E. Oliveira 1888 (IAN).

\section{AgRADECIMENTOS}

Ao Prof. José Maria Albuquerque, Faculdade de Ciências Agrárias do Pará, pela revisão da diagnose latina.

\section{SUMMARY}

In this paper the author presents notes about three species of genus Vismia Vandelli (Guttiferae): V. cayennensis (Jacquin) Persoon, V. subcuneata Huber and V. cavalcantii van den Berg, including the new variety $\mathbf{V}$. cayennensis var. tenuinervia van den Berg and the new combination $\mathbf{V}$. cayennensis var. zessilifolia (Aublet) van den Berg.

The validity of $\mathbf{V}$. subcuneata as a species is discuted here.

A complemental description of fruits of $\mathbf{v}$. ea. valcantii var. cavalcantii is included.

\section{BIBLIOGRAFIA CITADA}

EWAN, J.

1962 - Synopsis of the South American species of Vismia (Guttiferae). Contrib. U. S. Nat. Herb., 35(5):293-377.

HUBER, J.

1906 - Materiaes para a Flora Amazônica. B. Mus. Pa. Emílio Goeldi, Belém, 4:588.

REICHARDT, H. W.

1878 - Hypericaceae. In: Martius, Eichler \& Urban - "Flora Brasiliensis". Leipzig, Fried Fleischer Comp., 12(1):382-474.

STAFLEU, F. A. ET ALII ED.

1972 - International Code of Botanical Nomenclature, adapted by 11 th International Botanical Congress, Seattle, August 1969. Utrecht, Netherlands. $426 \mathrm{p}$.

VAN DEN BERG, M. E.

1970 - Uma espécie e duas variedades novas de Guttiferae da Amazônia. B. Mus. Pa. Emílio Goeldi, Belém, n. sér.: Botânica $38,6 \mathrm{p}$. 